HIV

\section{Silencing HIV the natural way}

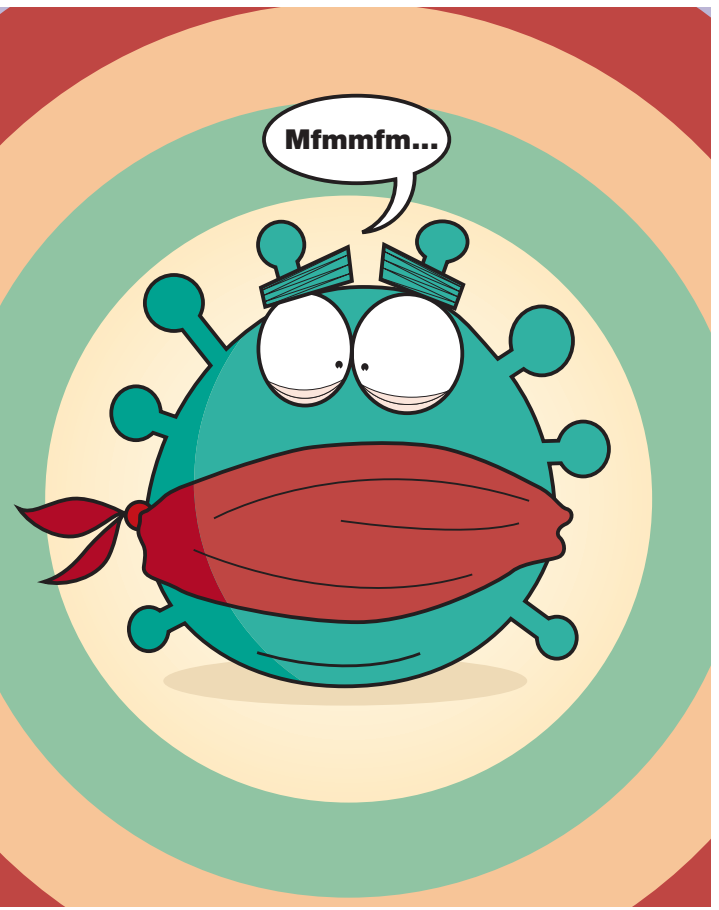

A recent paper in Immunity describes the first natural example of RNA silencing in mammalian host-virus interactions. This evolutionarily ancient form of nucleic-acid-based immunity has previously been found in plants and invertebrates. But the demonstration that human cells can physiologically silence HIV-1 RNA highlights the universal importance of this system.

RNA silencing (also known as RNA interference, RNAi) in invertebrates is based on the cleavage of double-stranded RNA precursors by the RNase Dicer to form short interfering RNAs (siRNAs) of $\sim 21$ nucleotides; siRNAs then induce the degradation of complementary target RNAs, leading to post-transcriptional gene silencing. Silencing of viral genes is a natural antiviral response in plants and insects, using siRNA derived from the viral genome by the host processing machinery.

To examine the relevance of this response in immunity to HIV-1, the authors looked for sequences in the viral genome that could form the RNA-duplex precursors necessary for cleavage by host Dicer. They found one sequence in HIV-1, in the env gene (which encodes the envelope surface glycoprotein), that formed a 19-base-pair duplex, and this sequence was functionally recognized by Dicer in vitro. Furthermore, the Dicer cleavage product - viral siRNA (vsiRNA) - was detected in HIV-infected T cells but not in mock-infected cells.

To test whether the vsiRNA could mediate RNAi, they used constructs of its target RNA sequence fused to enhanced green fluorescent protein (EGFP) such that cleavage of the target RNA would prevent transcription of the downstream EGFP-encoding RNA. The addition of vsiRNA precursors led to a dose-dependent silencing of EGFP expression, and these precursors could also silence expression of the cognate target HIV-1 env mRNA.

Because HIV-1 successfully replicates in human cells to high levels of cell-free RNA, it must be able to defend itself against this hostexploited RNAi. Synthetic siRNA precursors specific for the HIV-1 transactivation response element (TAR) were ineffective at suppressing EGFP expression from a TAR-EGFP transcript when the HIV-1 transcriptional transactivator (Tat) was included in the assay, and the suppressive function of Tat was also shown for other non-TAR sequences. This indicates that Tat is a general suppressor of RNA silencing (SRS). This

\title{
Homing in on the target
}

Although multiple pancreatic islet-cell molecules are targets of autoimmune responses in human type 1 diabetes and in mouse models, conclusive evidence of the autoantigens that are important for initiation of the disease has been difficult to obtain. Now, two articles in Nature show that insulin is likely to be a crucial autoantigen in the development of type 1 diabetes in both the mouse model and human patients.

Previously, it has been shown that most $\mathrm{CD}^{+} \mathrm{T}$ cells infiltrating the pancreas in non-obese diabetic (NOD) mice recognize insulin, in particular peptide 9-23 of the insulin B chain. So, to test the role of this response in the development of disease, Nakayama et al. generated NOD mice in which both insulin genes (Ins1 and Ins2) were deleted and replaced with a transgene encoding mutant pro-insulin. The mutant pro-insulin contained a single amino-acid change at position 16 of the insulin B chain, which preserves the metabolic activity of insulin but prevents recognition by the infiltrating T cells. None of the Ins $1^{-1-}$ Ins $2^{-1-}$ NOD mice expressing the modified insulin showed signs of an immune response to the islet cells, and they did not develop diabetes (although autoimmune reactions were still evident in the salivary glands). By contrast, the presence of either insulin gene in these mice was sufficient to restore diabetes, confirming a crucial role for both insulin genes as targets of organ-specific autoimmunity.

Unlike mouse studies, the study of human autoimmune diseases is often hindered by the scarcity and lability of the relevant target tissues or draining lymph nodes. But in the second study, Kent et al. obtained viable pancreatic draining lymph nodes from three individuals with type 1 diabetes (two with long-term disease and one with recent-onset disease) and three control individuals. From these lymphnode samples, the authors generated single
T-cell clones in a non-biased manner and analysed their T-cell receptor (TCR) repertoire and their antigen specificity. T-cell clones isolated from pancreatic lymph nodes of control individuals expressed heterogeneous TCR repertoires, indicative of polyclonal expansion. By contrast, more than half of the T-cell clones from the long-term diabetic individuals expressed identical $\mathrm{V} \beta$ chains, and of these clones, half had the same TCR $\alpha$-chain, implying antigen-driven expansion of a common progenitor cell. The clones from the long-term diabetic individuals, but not those from control individuals, proliferated specifically in response to peptide 1-15 of insulin A in a dosedependent manner. This response was restricted by the MHC class II allele HLA-DRB1 ${ }^{\star} 0401$, which is known to confer genetic susceptibility to diabetes.

To rule out the possibility that the insulinspecific $\mathrm{T}$-cell responses could have arisen from long-term use of daily insulin injections (to control blood glucose levels), Kent et al. showed that insulin-reactive T cells were not found in the spleen of one of the long-term diabetic individuals, and $\mathrm{CD}_{4}^{+} \mathrm{T}$-cell clones from pancreatic draining lymph nodes of 
